\title{
Antioxidant status in patients with sleep apnoea and impact of continuous positive airway pressure treatment
}

\author{
A. Barceló*, F. Barbé\#, M. de la Peña*, M. Vila*, G. Pérez*, J. Piérola*, \\ J. Durán ${ }^{\top}$ and A.G.N. Agustí ${ }^{+}$
}

ABSTRACT: The episodes of hypoxia/re-oxygenation associated with the respiratory disturbances observed in patients with obstructive sleep apnoea syndrome (OSAS) may induce the generation of oxygen free radicals. Indeed, several studies suggest that OSAS is associated with oxidative stress. The present study tested the hypothesis that patients with OSAS have an alteration in antioxidant defences.

The plasma levels of total antioxidant status (TAS), glutathione peroxidase (GPX), $\gamma$ glutamyltransferase (GGT), vitamins $A, E, B_{12}$ and folate, and homocysteine were determined in 47 patients with OSAS and 37 healthy subjects. Of these, 27 patients who used continuous positive airway pressure (CPAP) for $>4 \mathrm{~h} \cdot \mathrm{night}^{-1}$ were re-examined 12 months later.

Patients with OSAS had lower TAS $\left(1.4 \pm 0.16\right.$ versus $\left.1.50 \pm 0.10 \mathrm{mmol} \cdot \mathrm{L}^{-1}\right)$, vitamin A $(64 \pm 19$ versus $\left.74 \pm 17 \mu \mathrm{g} \cdot \mathrm{dL}^{-1}\right)$ and vitamin $E$ levels $\left(1,525 \pm 499\right.$ versus $\left.1,774 \pm 503 \mu \mathrm{g} \cdot \mathrm{dL}^{-1}\right)$, and increased values of GGT $\left(42 \pm 22\right.$ versus $\left.32 \pm 16 \mathrm{U} \cdot \mathrm{L}^{-1}\right)$ than controls. There was no difference between groups in GPX, homocysteine, vitamin $\mathrm{B}_{12}$ and folate plasma levels. CPAP treatment normalised the levels of TAS $\left(1.50 \pm 0.13 \mathrm{mmol} \cdot \mathrm{L}^{-1}\right)$ and the activity of GGT $\left(30 \pm 14 \mathrm{U} \cdot \mathrm{L}^{-1}\right)$ without any influence on vitamins levels.

In conclusion, the results indicate that patients with obstructive sleep apnoea syndrome have a decreased antioxidant capacity that is partially reversed by continuous positive airway pressure treatment.

KEYWORDS: Clinical trial, continuous positive airway pressure, oxidative stress

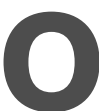
bstructive sleep apnoea syndrome (OSAS) is a common disorder characterised by the occurrence of numerous episodes of absence of respiratory flow (apnoea) during sleep, which can be followed by a decrease in arterial oxygen saturation $\left(\mathrm{Sa}_{1} \mathrm{O}_{2}\right)$ that is rapidly normalised when ventilation resumes. These episodes of hypoxia/re-oxygenation may induce the generation of oxygen free radicals [1]. In fact, several studies support the existence of oxidative stress in OSAS, and several authors have suggested a possible involvement of the latter in the pathogenesis of cardiovascular disease in these patients [2-4].

Oxidative stress is characterised by an imbalance between the production and degradation of reactive oxygen species. Antioxidant enzymes and vitamins counteract reactive oxygen species and potentially reduce their cellular damage [5]. The role of these protective mechanisms is

For editorial comments see page 671 . poorly explored in OSAS patients. In the present study, the authors hypothesised that antioxidant defences might be altered in OSAS. To test this hypothesis, the authors compared plasma total antioxidant status (TAS), the activity of antioxidant enzymes (glutathione peroxidases (GPX) and $\gamma$-glutamyl transferase (GGT)), antioxidant vitamins (A, E, $\mathrm{B}_{12}$ and folate) and homocysteine levels in patients with OSAS and healthy subjects. Furthermore, to assess the reversibility of potential abnormalities once the apnoeic events had been eliminated by the use of continuous positive airway pressure (CPAP) therapy, these same variables were determined again after 12 months of treatment with CPAP.

\section{METHODS}

\section{Subjects and ethics}

In total, 47 male patients with OSAS and 37 healthy control subjects were studied. The diagnosis of OSAS was established by full polysomnography (Ultrason Nicolett, WI, USA) and included recordings of the following: 1) oronasal

\section{AFFILIATIONS}

*Serveis de Anàlisis Cliniques, and

\#Pneumologia, Hospital Universitario Arnau de Vilanova, Lleida,

"Hospital Txagorritxu,

Vitoria-Gasteiz, and

${ }^{+}$Hospital Universitari, Son Dureta,

Institute Universitari de Ciencies de la Salut (IUNICS), Fundació Caubet-

Cimera, Palma de Mallorca and

Servicio de Neumología, Spain.

CORRESPONDENCE

A. Barceló

Servicio de Análisis Clínicos

Hospital

Universitari Son Dureta. C/ Andrea

Doria 55

07014 Palma de Mallorca

Spain

Fax: 34971175228

E-mail: abarcelo@hsd.es

Received:

June 102005

Accepted after revision:

November 252005

SUPPORT STATEMENT

This study was supported in part by ABEMAR, SEPAR and Fondo de Investigaciones Sanitarias (Red Respira (RTIC C03/11), 02/0334, 04/ 1593 and expte. CM-0300049†). 
flow; 2) thoracoabdominal movements; 3) electrocardiography; 4) submental and pretibial electromyography; 5) electrooculography; 6) electroencephalography; and 7) trancutaneous measurement of $\mathrm{Sa}_{2} \mathrm{O}_{2}$. Apnoea was defined by the absence of airflow for $>10 \mathrm{~s}$. Hypopnoea was defined as any airflow reduction that lasts $>10 \mathrm{~s}$ and resulted in arousal or oxygen desaturation. Desaturation was considered a decrease in $\mathrm{Sa}_{1} \mathrm{O}_{2}$ of $>4 \%$. The apnoea-hypopnoea index (AHI) was defined as the sum of the number of apnoeas plus hypopnoeas per hour of sleep. Arterial hypertension was diagnosed if systolic blood pressure (SBP) was $>140 \mathrm{mmHg}$ or diastolic pressure (DBP) was $>90 \mathrm{mmHg}$. Patients were studied at diagnosis and after having been treated effectively with CPAP (REM Star; Respironics ${ }^{\circledR}$, Murrysville, PA, USA) during 12 months. Compliance with treatment was checked by the timer built up in the CPAP device. A priori, good compliance for posttreatment evaluation was defined as a mean usage of CPAP $>4 \mathrm{~h} \cdot$ night $^{-1}$. According to these criteria, 20 patients were excluded from the follow-up analysis.

Control subjects were healthy nonsmoker males of similar age and body mass index (BMI) than patients with OSAS. They had no personal or family history of cardiovascular disease or diabetes. Nonobese controls $(n=17)$ were nonsnorers recruited from nonmedical staff of the authors' hospital. In these subjects, the diagnosis of OSAS was excluded by a cardiorespiratory sleep study that recorded nasal flow, thoracic movements, heart rate, snoring, body position and transcutaneus oxyhaemoglobin saturation (Edentec, Eden Prairie, MN, USA). Obese controls (BMI $>30 \mathrm{~kg} \cdot \mathrm{m}^{-2} ; \mathrm{n}=20$ ) were recruited from obese subjects who attended the authors' sleep unit, in whom OSAS was excluded by full polysomnography. No participant (patient or control) suffered from any chronic disease (chronic obstructive pulmonary disease, diabetes mellitus, liver cirrhosis, thyroid dysfunction, rheumatoid arthritis, chronic renal failure and/or psychiatric disorders), or was taking any type of medication. The study was approved by the Ethics Committee of the authors' institution. All participants signed their consent after being fully informed of the goal and characteristics of the study.

\section{Measurements}

After fasting overnight, venous blood samples were obtained between 08:00-10:00 h. Blood was centrifuged and serum was immediately separated in aliquots and stored at $-80^{\circ} \mathrm{C}$ until analysis. Glucose, uric acid, triglycerides and cholesterol concentrations were determined by standard enzymatic methods on a Hitachi 917 biochemical analyser (Roche Diagnostics, Indianapolis, IN, USA). High-density lipoprotein cholesterol (HDLc) was measured by a homogeneous, enzymatic colorimetric method using a commercial reagent set (Roche Diagnostics, USA). Low-density lipoprotein cholesterol was calculated using the Friedewald equation. The plasma concentration of TAS was measured by a colorimetric test (Randox laboratories Ltd, Crumlin, UK). GPX and GGT activities were determined by enzymatic methods (Randox laboratories Ltd and Roche Diagnostics, Manheim, Germany, respectively) on a Hitachi Modular Analytics system (Roche Diagnostics, Germany). The plasma levels of vitamin A and E were measured by HPLC following the manufacturers' instructions (Bio Rad laboratories $\mathrm{GmbH}$, Munich, Germany).
The plasma concentrations of vitamin $\mathrm{B}_{12}$, folate and homocysteine were quantified by chemiluminiscent assays on an Immulite 2000 analyzer (DPC, Los Angeles, CA, USA; vitamin $\mathrm{B}_{12}$ and folate) or Advia Centaur analyser (Bayer, Tarrytown, NY, USA).

\section{Statistical analysis}

Data are presented as mean \pm SD. Comparisons between patients and controls were performed with independent t-tests and Chi-squared tests for proportions. The effects of CPAP therapy were analysed using paired t-tests. The correlation between variables was explored using the Spearman test. A p-value $<0.05$ was considered significant.

\section{RESULTS \\ Baseline data}

Table 1 shows the main clinical characteristics of patients with OSAS and healthy controls. Age and BMI were similar. Patients showed significantly higher mean values of diastolic blood pressure than controls. The number of hypertensive subjects and current smokers was higher in the patient group. Patients had severe OSAS (as shown by their AHI) and reported daytime sleepiness (Epworth sleep scale).

Table 2 reports the values of the different biological markers investigated in the present study. In patients with OSAS, the levels of HDLc were significantly lower than in healthy controls. Furthermore, OSAS patients had lower TAS, vitamin A and E levels than controls. Also, increased values of GGT were detected in the OSAS group. The OSAS and control groups did not differ significantly in GPX, homocysteine, vitamin $B_{12}$ and folate plasma levels.

To evaluate the potential confounding effect of active smoking, the study variables in nonsmoker patients and nonsmoker controls were compared. It was observed that after excluding smokers the main results did not change (table 3). Furthermore, the authors compared these variables between smoker and nonsmoker patients and found that the former had slightly higher levels of homocysteine than the latter $(12.4 \pm 6.0$

\begin{tabular}{|c|c|c|}
\hline \multirow[t]{2}{*}{ TABLE 1} & \multicolumn{2}{|c|}{$\begin{array}{l}\text { Clinical characteristics of patients and healthy } \\
\text { subjects }\end{array}$} \\
\hline & Controls & OSAS \\
\hline Subjects $n$ & 37 & 47 \\
\hline Age yrs & $46 \pm 9$ & $48 \pm 9$ \\
\hline BMI $\mathbf{k g} \cdot \mathrm{m}^{-2}$ & $29.6 \pm 5.0$ & $30.2 \pm 5.2$ \\
\hline SBP mmHg & $128 \pm 12$ & $132 \pm 16$ \\
\hline DBP $\mathrm{mmHg}$ & $77 \pm 8$ & $85 \pm 10^{* \star *}$ \\
\hline Current smokers \% & 0 & $19(40)^{\star \star \star}$ \\
\hline Hypertension \% & 0 & $15(31)^{\star \star \star}$ \\
\hline AHI events $\cdot h^{-1}$ & $2 \pm 1$ & $49 \pm 16^{\star \star \star}$ \\
\hline Mean sat $\mathrm{O}_{2} \%$ & $93 \pm 1.6$ & $92 \pm 2.7$ \\
\hline Epworth scale & $3 \pm 1$ & $13 \pm 6^{\star * *}$ \\
\hline \multicolumn{3}{|c|}{$\begin{array}{l}\text { Data are presented as mean } \pm \mathrm{SD}, \mathrm{n} \text { or } \mathrm{n}(\%) \text {. OSAS: obstructive sleep apnoea } \\
\text { syndrome; BMI: body mass index; SBP: systolic blood pressure; DBP: diastolic } \\
\text { blood pressure; AHI: apnoea-hypopnoea index. }{ }^{\star \star \star}: \mathrm{p}<0.001 \text { versus healthy } \\
\text { controls. }\end{array}$} \\
\hline
\end{tabular}




\begin{tabular}{|c|c|c|c|}
\hline \multirow[t]{2}{*}{ TABLE 2} & \multicolumn{3}{|c|}{$\begin{array}{l}\text { Results at baseline between control subjects and } \\
\text { patients with obstructive sleep apnoea syndrome } \\
\text { (OSAS) }\end{array}$} \\
\hline & & Controls & OSAS \\
\hline \multicolumn{2}{|l|}{ Subjects n } & 37 & 47 \\
\hline \multicolumn{2}{|c|}{ Glucose $\mathrm{mg} \cdot \mathrm{dL}^{-1}$} & $105 \pm 10$ & $109 \pm 19$ \\
\hline \multicolumn{2}{|c|}{ Triglycerides $\mathbf{m g} \cdot \mathrm{dL}^{-1}$} & $147 \pm 92$ & $143 \pm 96$ \\
\hline \multicolumn{2}{|c|}{ Cholesterol mg $\cdot \mathrm{dL}^{-1}$} & $223 \pm 30$ & $220 \pm 33$ \\
\hline \multicolumn{2}{|c|}{$\mathrm{LDLc} \mathbf{m g} \cdot \mathrm{dL}^{-1}$} & $150 \pm 34$ & $148 \pm 30$ \\
\hline \multicolumn{2}{|c|}{$\mathrm{HDLc} \mathbf{m g} \cdot \mathrm{dL}^{-1}$} & $51 \pm 9$ & $45 \pm 9^{\#}$ \\
\hline \multicolumn{2}{|c|}{ Uric acid $\mathbf{m g} \cdot \mathrm{dL}^{-1}$} & $6.6 \pm 1.2$ & $6.9 \pm 1.3$ \\
\hline \multicolumn{2}{|c|}{ TAS $\mathrm{mmol} \cdot \mathrm{L}^{-1}$} & $1.50 \pm 0.10$ & $1.40 \pm 0.16^{\star \star *}$ \\
\hline \multicolumn{2}{|c|}{ GGT U. $L^{-1}$} & $32 \pm 16$ & $42 \pm 22^{\star}$ \\
\hline \multicolumn{2}{|l|}{ GPX U. $L^{-1}$} & $798 \pm 19$ & $844 \pm 19$ \\
\hline \multicolumn{2}{|c|}{ Vitamin $A \mu \mathbf{g} \cdot \mathrm{dL}^{-1}$} & $74 \pm 17$ & $64 \pm 19^{*}$ \\
\hline \multicolumn{2}{|c|}{ Vitamin $\mathrm{E} \mu \mathrm{g} \cdot \mathrm{dL}^{-1}$} & $1774 \pm 503$ & $1525 \pm 499^{*}$ \\
\hline \multicolumn{2}{|c|}{ Vitamin $B_{12} \mathrm{pg} \cdot \mathrm{mL}^{-1}$} & $475 \pm 312$ & $501 \pm 352$ \\
\hline \multicolumn{2}{|c|}{ Folate $\mathbf{n g} \cdot \mathrm{mL}^{-1}$} & $9.5 \pm 3.5$ & $10.0 \pm 3.3$ \\
\hline \multicolumn{2}{|c|}{$\begin{array}{l}\text { Homocysteine } \\
\mu \mathrm{mol} \cdot \mathrm{L}^{-1}\end{array}$} & $11.8 \pm 4.2$ & $10.8 \pm 4.3$ \\
\hline
\end{tabular}

Data are presented as mean \pm SD unless otherwise stated. LDLC: low-density lipoprotein cholesterol; HDLC: high-density lipoprotein cholesterol; TAS: total antioxidant status; GGT: $\gamma$-glutamyltransferase; GPX: glutathione peroxidase. ${ }^{\#}$ : $p<0.005 ; *: p<0.05 ; * * *: p<0.001$ versus healthy controls.

\begin{tabular}{|c|c|c|}
\hline \multirow[t]{2}{*}{ TABLE 3} & \multicolumn{2}{|c|}{$\begin{array}{l}\text { Results at baseline of control subjects and } \\
\text { nonsmoker patients with obstructive sleep } \\
\text { apnoea syndrome }\end{array}$} \\
\hline & Controls & Nonsmokers \\
\hline Subjects $n$ & 37 & 28 \\
\hline HDLc $\mathbf{m g} \cdot \mathrm{dL}^{-1}$ & $51 \pm 9$ & $46 \pm 9^{*}$ \\
\hline TAS $\mathrm{mmol} \cdot \mathrm{L}^{-1}$ & $1.50 \pm 0.13$ & $1.41 \pm 0.14^{\star}$ \\
\hline GGT U.L ${ }^{-1}$ & $32 \pm 16$ & $41 \pm 17^{\star}$ \\
\hline Vitamin $\mathbf{A} \boldsymbol{\mu g} \cdot \mathrm{dL}^{-1}$ & $74 \pm 17$ & $64 \pm 21^{*}$ \\
\hline Vitamin $\mathrm{E} \boldsymbol{\mu \mathrm { g }} \cdot \mathrm{dL}^{-1}$ & $1774 \pm 503$ & $1550 \pm 434^{\star}$ \\
\hline
\end{tabular}

Data are presented as mean $\pm S D$ or $n$. HDLc: high-density lipoprotein cholesterol; TAS: total antioxidant status; GGT: $\gamma$-glutamyltransferase. *: $p<0.05$ versus healthy controls

versus $\left.9.8 \pm 2.1 \mu \mathrm{mol} \cdot \mathrm{L}^{-1} ; \mathrm{p}=0.04\right)$. By contrast, there were no significant differences in vitamins or enzyme plasma levels. Finally, the presence of hypertension had no effect on the parameters studied because similar results were observed in hypertensive and normotensive patients (data not shown).

\section{Effects of CPAP treatment}

In total, 27 patients used CPAP for $>4 \mathrm{~h} \cdot$ night $^{-1}(5.7 \pm$ $1.4 \mathrm{~h} \cdot$ night $^{-1}$ ) and were re-assessed after 12 months of treatment. There was no difference in the baseline characteristics shown in table 1 between compliant and noncompliant patients. BMI did not change after CPAP therapy (30.0 versus $\left.30.2 \mathrm{~kg} \cdot \mathrm{m}^{-2} ; \mathrm{p}=0.4\right)$. The plasma levels of HDLc and TAS were
TABLE 4 Effects of continuous positive airway pressure (CPAP) treatment

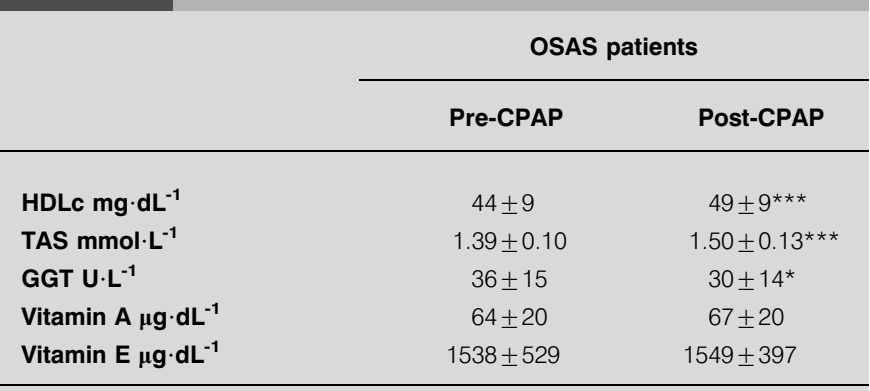

Data are presented as mean \pm SD. HDLc: high-density lipoprotein cholesterol TAS: total antioxidant status; GGT: $\gamma$-glutamyltransferase. $n=27$; *: $p<0.05$; ***: $p<0.001$ versus pre-CPAP

significantly higher and those of GGT significantly lower than those determined at baseline. Interestingly, values after CPAP were not different from those of the control group. In contrast, CPAP did not influence vitamins and homocysteine levels (table 4).

No significant correlation was detected between the main variables studied (TAS, vitamins and antioxidant enzymes) and AHI, nocturnal mean $\mathrm{Sa}_{2} \mathrm{O}_{2}, \mathrm{SBP}, \mathrm{DBP}$ and the Epworth sleep scale at baseline (data not shown).

\section{DISCUSSION}

The main findings of the present study were as follows: 1) Patients with OSAS showed reduced antioxidant capacity compared with healthy controls, as reflected by their lower TAS, vitamin A and E levels as well as by their higher GGT activity. 2) Chronic treatment with CPAP partially improves antioxidant defence, as shown by the increase in TAS levels and the decrease in GGT activity after CPAP.

The results of several studies, including the present study, support the presence of oxidative stress in obstructive sleep apnoea $[2-4,6,7]$. In keeping with this argument, the current authors found that the patients with OSAS had lower TAS values than controls. A negative correlation between antioxidant capacity and AHI has been previously detected in patients with OSAS (AHI >20) [6]. This observation could not be reproduced, probably because of the narrow range of disease severity in the study patients. The fact that TAS was normalised after CPAP therapy, known to improve other indices of oxidative stress in OSAS [3,4], suggests that the low TAS observed in patients with OSAS at baseline is probably the consequence, not the cause, of oxidative stress in these patients.

Previous studies support the argument that serum GGT activity is a marker of oxidative stress [7]. The primary function of GGT is to maintain intracellular concentrations of glutathione, a critical antioxidant molecule [8]. Thus, an increased GGT activity can also be viewed as a response to oxidative stress, aimed at increasing the intracellular concentration of glutathione [8]. This interpretation is further supported by the significant reduction of GGT activity after CPAP therapy. Emerging evidence shows that serum GGT 
might be important in the pathogenesis of oxidant-related cardiovascular disease [9]. Hence, increased serum GGT activity in OSAS may identify patients with persistent oxidative stress and increased cardiovascular risk. Increased plasma levels of GGT have also been described in obese subjects [9]. In the current study, the BMI of patients with OSAS was similar to that of controls (table 1), thus, excluding a significant role of obesity upon the increased GGT activity seen in OSAS. Furthermore, the decrease of GGT detected after CPAP treatment, without any change in BMI, supports the hypothesis that this increase is linked directly to OSAS.

HDLc is one of the most important protective factors for atherosclerosis [10, 11]. The levels of HDLc were found to be significantly lower in OSAS than in controls, and they increased significantly after CPAP treatment. Likewise, OSAS patients were found to have lower levels of vitamin A and E than control subjects. These vitamins are considered lipid-soluble antioxidants as they can prevent oxidative damage of lipid membranes and low density lipoproteins $[12,13]$. Therefore, overall these findings highlight potential mechanisms for increased lipid peroxidation and enhanced atherosclerosis risk in OSAS, as well as a potentially beneficial effect of CPAP in these patients.

A recent prospective study has reported that low erythrocyte glutathione peroxidase-1 activity identifies patients with coronary artery disease with the highest risk of acute cardiovascular events [14]. The authors suggested that this can be a useful marker to identify patients who would benefit most from preventive antioxidant treatment [14]. In the present study, significant differences in GPX activity between OSAS and controls were not detected. However, the intracellular activity of GPX was not measured, which may limit the interpretation of the present results.

Homocysteine is emerging as an independent risk factor for cardiovascular disease $[15,16]$, but it is not clear if homocysteine is associated with sleep apnoea. LAVIE et al. [2] showed that patients with a combination of OSAS and ischaemic heart disease have elevated homocysteine levels. By contrast, the results of another study suggest that OSAS is not associated with raised homocysteine levels [17]. There were no significant differences in vitamin $B_{12}$, folate and homocysteine levels between OSAS and controls. Cigarette smoking is known to influence homocysteine levels and it is a possible confounder in the relationship between homocysteine and cardiovascular disease [18]. In fact, in the present study, OSAS patients who smoked showed higher homocysteine levels than nonsmokers. Yet, a potential influence of smoking on other variables can be excluded because differences between smoker versus nonsmoker patients, and between control subjects and nonsmoker patients, were not significant.

Previous studies have demonstrated a beneficial effect of CPAP on several markers of oxidative stress in patients with OSAS $[3,19]$. As oxidative stress is likely to play an important role in the increased cardiovascular risk that characterises this disease, these observations support a potential effect of CPAP in the prevention of cardiovascular complications in OSAS. In keeping with this view, it was found that chronic treatment with CPAP improves TAS, further supporting a potential beneficial effect of CPAP on oxidative stress in these patients. However, these effects must be taken with caution because the current study was not a randomised, controlled trial.

In conclusion, the present results indicate that antioxidant capacity is decreased in patients with obstructive sleep apnoea syndrome and that it is partially restored by continuous positive airway pressure treatment. These observations may be relevant for a better understanding of the pathogenesis of cardiovascular disorders in these patients.

\section{REFERENCES}

1 Douglas NJ, Polo O. Pathogenesis of sleep apnoea/ hypopnoea syndrome. Lancet 1994; 344: 653-655.

2 Lavie L. Obstructive sleep apnoea syndrome: an oxidative stress disorder. Sleep Med Rev 2003; 7: 35-51.

3 Schulz R, Mahmoudi S, Hattar K, et al. Enhanced release of superoxide from polymorphonuclear neutrophils in obstructive sleep apnea. Impact of continuous positive airway pressure therapy. Am J Respir Crit Care Med 2000; 162: 566-570.

4 Barcelo A, Miralles C, Barbe F, Vila M, Pons S, Agusti AGN. Abnormal lipid peroxidation in patients with sleep apnea. Eur Respir J 2000; 16: 644-647.

5 Griendling KK, Fitzgerald GA. Oxidative stress and cardiovascular injury. Part I: basic mechanisms and in vivo monitoring of ROS. Circulation 2003; 108: 1912-1916.

6 Christou K, Moulas AN, Pastaka C, Gourgoulianis KI. Antioxidant capacity in obstructive sleep apnea patients. Sleep Med 2003; 4: 225-228.

7 Lee DH, Gross MD, Jacobs JRDR. Association of serum carotenoids and tocopherols with $\gamma$-glutamyltransferase: the cardiovascular risk development in young adults (CARDIA) study. Clin Chem 2004; 50: 582-588.

8 Whitfield JB. Gamma glutamyl transferase. Crit Rev Clin Lab Sci 2001; 38: 263-355.

9 Lee DH, Jacobs JRDR, Gross M, et al. Gamma-glutamyltrannsferase is a predictor of incident diabetes and hypertension: the coronary artery risk development in young adults (CARDIA) study. Clin Chem 2003; 49: 1358-1366.

10 Gordon T, Castelli WP, Hjortland MC, Kannel WB, Dawber TR. High density lipoprotein as a protective factor against coronary heart disease. The Framingham study. Am J Med 1977; 62: 707-714.

11 Gordon DJ, Probstfield JL, Garrison RJ, Neaton JD, Castelli WP, Knoke JD. High density lipoprotein cholesterol and cardiovascular disease. Four prospective American studies. Circulation 1989; 79: 8-15.

12 Fairfield KM, Fletcher RH. Vitamins for chronic disease prevention in adults. JAMA 2002; 287: 3116-3126.

13 Steiner M. Vitamin E: more than an antioxidant. Clin Cardiol 1993; 16: I16-I18.

14 Blankenberg S, Rupprecht HJ, Bickel C, et al. Glutathione peroxidase 1 activity and cardiovascular events in patients with coronary artery disease. N Engl J Med 2003; 349: 1605-1613.

15 Mangoni AA, Jackson SH. Homocysteine and cardiovascular disease: current evidence and future prospects. Am J Med 2002; 112: 556-565. 
16 Clarke R, Stansbie D. Assessment of homocysteine as a cardiovascular risk factor in clinical practice. Ann Clin Biochem 2001; 38: 624-632.

17 Robinson GV, Pepperell JCT, Segal HC, Davies RJO, Stradling JR. Circulating cardiovascular risk factors in obstructive sleep apnoea: data from randomised controlled trials. Thorax 2004; 59: 777-782.

18 Sobczak A, Wardas W, Zielinska-Danch W, Pawlicki K. The influence of smoking on plasma homocysteine and cysteine levels in passive and active smokers. Clin Chem Lab Med 2004; 42: 408-414.

19 Carpagnano GE, Kharitonov SA, Resta O, FoschinoBarbaro MP, Gramiccioni E, Barnes PJ. 8-Isoprostane, a marker of oxidative stress, is increased in exhaled breath condensate of patients with obstructive sleep apnea after night and is reduced by continuous positive airway pressure therapy. Chest 2003; 124: 1386-1392. 